\title{
Antiferromagnetism in hexagonal graphene structures: Rings versus dots
}

\author{
M. Grujić, ${ }^{1, *}$ M. Tadić,,${ }^{1, \dagger}$ and F. M. Peeters ${ }^{2, \ddagger}$ \\ ${ }^{1}$ School of Electrical Engineering, University of Belgrade, P.O. Box 3554, 11120 Belgrade, Serbia \\ ${ }^{2}$ Department of Physics, University of Antwerp, Groenenborgerlaan 171, B-2020 Antwerp, Belgium \\ (Received 19 November 2012; revised manuscript received 27 January 2013; published 20 February 2013)
}

\begin{abstract}
The mean-field Hubbard model is used to investigate the formation of the antiferromagnetic phase in hexagonal graphene rings with inner zigzag edges. The outer edge of the ring was taken to be either zigzag or armchair, and we found that both types of structures can have a larger antiferromagnetic interaction as compared with hexagonal dots. This difference could be partially ascribed to the larger number of zigzag edges per unit area in rings than in dots. Furthermore, edge states localized on the inner ring edge are found to hybridize differently than the edge states of dots, which results in important differences in the magnetism of graphene rings and dots. The largest staggered magnetization is found when the outer edge has a zigzag shape. However, narrow rings with armchair outer edge are found to have larger staggered magnetization than zigzag hexagons. The edge defects are shown to have the least effect on magnetization when the outer ring edge is armchair shaped.
\end{abstract}

DOI: 10.1103/PhysRevB.87.085434

PACS number(s): 75.75.-c, 73.22.Pr, 81.05.ue

Recent tremendous progress in graphene research is driven by its remarkable properties, e.g., high crystalline quality, high electron mobility, lack of a band gap, and a minimal possible thickness, to name a few. ${ }^{1}$ The mentioned properties are advantageous for various applications of graphene, such as piezoelectric devices, ${ }^{2}$ supercapacitors, ${ }^{3}$ photodetectors, ${ }^{4}$ and field-effect transistors. ${ }^{5,6}$ Furthermore, it has been predicted that graphene structures could exhibit magnetic ordering which is potentially advantageous for spintronic applications. ${ }^{7,8}$ This effect is essentially related to either the global or local imbalance of sublattice atoms in bipartite lattices. An imbalance might give rise to zero-energy states in the electron spectrum. These states are localized near the zigzag edges or vacancies and, along with the repulsive electron-electron $(e-e)$ interaction, could eventually lead to a spin polarization of the ground state of the system. ${ }^{7}$ Furthermore, the spins on the same sublattice are found to exhibit ferromagnetic coupling along the graphene edges, whereas the spins on different sublattices along the graphene edges couple antiferromagnetically.

In theory, magnetic ordering has been demonstrated for graphene flakes, ${ }^{9}$ nanoribbons, ${ }^{10}$ and vacancies in bulk graphene. ${ }^{11}$ On the other hand, experimental reports on magnetism in graphene structures are rare and conflicting. They range from the detection of ferromagnetic or antiferromagnetic ordering ${ }^{12-14}$ to measurements of defect-induced paramagnetism. ${ }^{15,16}$ Magnetic ordering was even found to be preserved at room temperature. ${ }^{17,18}$ The essential cause of magnetism in graphene is the existence of a peak in the density of nonbonding edge states near the Fermi energy. However, due to the high reactivity of these states, magnetism might be strongly suppressed. ${ }^{19}$ Several theoretical studies offered explanations for a diversity of phenomena related to magnetic ordering and its suppression, which might occur by means of nonmagnetic edge passivation, edge reconstruction, or vanishing of spin correlations with increasing temperature. ${ }^{19,20}$ Hence, in order to experimentally detect magnetic ordering, graphene samples should be kept under rigorously controlled conditions. Yet, various applications of this effect have been proposed. They involve half-metallicity with electrically controlled spin propagation, ${ }^{8}$ defect-induced spin filtering, ${ }^{21}$ and spin logic devices. ${ }^{22,23}$

In this report we employ the mean-field Hubbard model to study the formation of local magnetic moments in hexagonal graphene rings. Our aim is to explore how magnetic ordering is affected by the ring size and the edge type. In order to identify different hexagonal rings, we introduce the following notation which might be visually aiding. We assume that the type of the inner ring edge is zigzag, and that $N$ unit hexagons are adjacent to this boundary. The outer ring edge is assumed to be comprised of either $M$ dimers if it is of armchair type, or $M$ unit hexagons if it is of zigzag type. Therefore, the ring is denoted by $M: N$. As an example, consider the ring shown in Fig. 1, which is assumed to be formed out of the hexagonal dot with armchair edge, which contains seven dimers at each side of the hexagon, as shown in Fig. 1(a). The ring is formed when the carbon atoms around the center of the dot are removed, as depicted in Figs. 1(a) and 1(b). Potentially, these exotic structures could be manufactured via substitutional doping of boron-nitride nanostructures with carbon. ${ }^{24}$ Because the edge of the removed dot has four unit hexagons at each side, the ring is denoted as $7_{A C}: 4_{Z G}$. The distributions of the magnetic moments in the graphene rings will be compared with the magnetic moment distributions in the hexagonal graphene dots. Those dots are assumed to have zigzag edges and are labeled by $N_{Z G}$, where $N$ has the same meaning as the symbol $M$ for the rings.

Magnetic ordering of a graphene structure is governed by Lieb's theorem. ${ }^{25}$ It states that the total ground-state spin of a bipartite lattice with repulsive $e$ - $e$ interaction as described by the Hubbard model equals half of the difference of the sublattice sites. For symmetrical structures, this rule is related to the arrangement of the carbon atoms with respect to lines of reflection symmetry in the graphene plane: if there is a symmetry line which does not intersect any of the carbon atoms, the total ground-state spin is zero; otherwise there exists a finite magnetic moment. All the hexagonal rings analyzed here possess such a symmetry, thus their total magnetization equals zero, unlike triangular rings which display a ferrimagnetic phase. ${ }^{26}$ However, Lieb's theorem does 


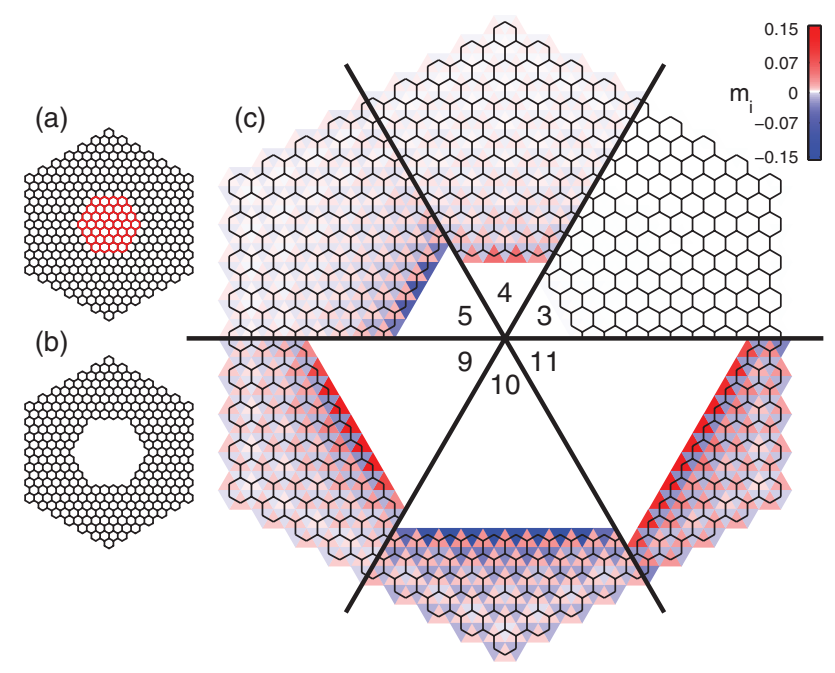

FIG. 1. (Color online) (a) The dot (red color) with four atoms at the zigzag edge removed from the larger dot (black color) which has seven dimers at the dot edge. (b) The formed ring is labeled by $7_{A C}: 4_{Z G}$. (c) The distribution of magnetic moments in the $9_{A C}: N_{Z G}$ ring shown in a sextant of the ring for $N$ taking the values 3, 4, 5, 9, 10 , and 11 . The majority spin is labeled by both orientation and color of a triangle centered at an atomic site. The local magnetic moment value is proportional to the color intensity.

not dictate the distribution of the local magnetic moments or the lack of zero-energy states. Furthermore, the number of zero-energy states in the analyzed ring is an integer multiple of six, which is a consequence of the $C_{6 v}$ symmetry of the ring. In the $9_{A C}: 10_{Z G}$ ring, six zero-energy states are found, which agrees with graph theory, and which is a topological property related to the nonperfect matching of the $p_{z}$ orbitals. $^{7}$

The Hubbard Hamiltonian

$$
H=H_{0}+H_{I}
$$

is employed to compute the distribution of magnetic moments. $H_{0}$ is the noninteracting part, which represents the nearestneighbor tight-binding Hamiltonian and is given by

$$
H_{0}=-t \sum_{\langle i, j\rangle, \sigma} c_{i \sigma}^{\dagger} c_{j \sigma},
$$

where $c_{j \sigma}$ and $c_{j \sigma}^{\dagger}$ are the annihilation and creation operators, respectively, and $t$ denotes the hopping integral. The interacting part $H_{I}$ describes the $e$ - $e$ interaction

$$
H_{I}=U \sum_{i}\left(n_{i \uparrow}\left\langle n_{i \downarrow}\right\rangle+n_{i \downarrow}\left\langle n_{i \uparrow}\right\rangle-\left\langle n_{i \uparrow}\right\rangle\left\langle n_{i \downarrow}\right\rangle\right),
$$

where $n_{i \sigma}=c_{i \sigma}^{\dagger} c_{i \sigma}$ is the number operator, and $U$ denotes the on-site Coulomb repulsion energy for each pair of electrons with the opposite spins orbiting the same atom. ${ }^{27}$ Equation (3) is obtained within the mean-field approximation, which assumes that the spin-up (spin-down) electrons interact with the average density of spin-down (spin-up) electrons on a particular atomic site.

In our calculations, we take $t=2.7 \mathrm{eV}$ and $U=1.2 t .^{7}$ We note that there is no consensus on the actual value of the strength of the Coulomb interaction to be used in the Hubbard model in graphene. Recent density functional theory
(DFT) calculations came up with a value closer to $U=3.4 t .^{28}$ However, having in mind that the mean-field approximation can overestimate the tendency for magnetic order for large $U{ }^{29}$ we chose the more conservative value of $U=1.2 t$. The solution is then obtained by means of a self-consistent procedure which starts from an initial distribution of the spins and ends when the maximum change of the electron density over the atomic sites drops below $10^{-5}$. When the self-consistent spin densities are determined, the magnetic moment per site $m_{i}$ is computed as

$$
m_{i}=\left\langle s_{i}^{z}\right\rangle=\left(\left\langle n_{i \uparrow}\right\rangle-\left\langle n_{i \downarrow}\right\rangle\right) / 2 .
$$

For the antiferromagnetic order parameter we take the staggered magnetization

$$
\mu_{s}^{z}=\frac{1}{N} \sum_{i}(-1)^{i}\left\langle s_{i}^{z}\right\rangle,
$$

where $(-1)^{i}$ symbolizes that we sum up the contributions from opposite sublattices with opposite signs. This is the appropriate order parameter for antiferromagnetism when examining spin polarization occurring in bipartite lattices. The larger $\mu_{s}^{z}$ is, the stronger is the antiferromagnetic phase. In addition to $\mu_{s}^{z}$, the shift in the electron and the hole energy spectra which arises from the magnetic order is quantified as $\Delta E=\left(E^{\mathrm{HOS}}+\right.$ $\left.E^{\text {LUS }}\right) / 2$, where $E^{\mathrm{HOS}}$ and $E^{\text {LUS }}$ are the highest-occupied and lowest-unoccupied states in the ground state at half filling, respectively. Note that in the nonmagnetic state we have $\Delta E=0$. We will explore how the maximum magnetic moment $m_{\max }$ varies with the ring width.

The distribution of the local magnetic moments in the $9_{A C}: N_{Z G}$ rings for several values of $N$ is shown in Fig. 1(c). The symmetry of each hexagonal ring is $C_{6 v}$, whereas the symmetry of the magnetic moment distribution is $I C_{6 v}$, i.e., the magnetic moments alter sign when rotated over $\pi / 3 \mathrm{rad}$. Therefore, it suffices to display the distribution of magnetic moments in sectors of $\pi / 3 \mathrm{rad}$, as done in Fig. 1(c), which combines the sectors of different $N$. Orientation and color of triangles denotes the orientation of the magnetic moments, and the absolute value of $m_{i}$ is depicted by color intensity.

It is evident in Fig. 1(c) that both the appearance of staggered magnetization and the total magnetic moment situated on the inner edge of the ring depend on the ring width. Furthermore, we observe a phase change from nonmagnetic order for $N \leqslant 3$ to antiferromagnetic order for $N \geqslant 4$, which is similar to previous calculations for zigzag hexagonal graphene dots. ${ }^{9,10}$ No magnetic ordering for zigzag segments shorter than three unit cells is found because of the close proximity of the opposite sublattice imbalance on the adjacent sides of the ring inner edge. When this edge is short, the edge states on the different sides of the inner ring boundary are subject to strong hybridization, and therefore their energy is lifted from the Dirac point. Hence, spontaneous spin polarization does not occur, which is similar to the case of nanoribbons. ${ }^{30}$

For $N \geqslant 4$, the spatial spin symmetry is broken due to the $e-e$ interaction. When the ring width decreases, the maximum magnetic moment, which is located near the middle of the zigzag edge segment increases. Furthermore, nonzero magnetization is built up on the outer ring edge, and it increases when the ring width decreases. However, as a consequence of the increasing influence of the outer edge with decreasing ring 


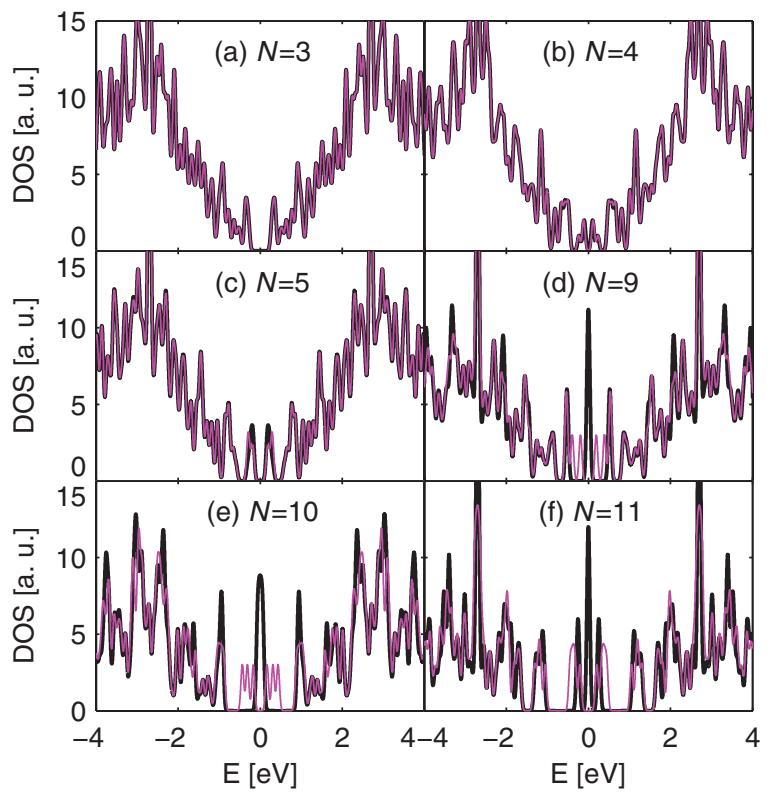

FIG. 2. (Color online) Density of states in the $9_{A C}: N_{Z G}$ rings for $N$ taking the values $3,4,5,9,10$, and 11 in the noninteracting system (black lines) and the interacting system (purple lines).

width, the difference between the distributions of the magnetic moments on the two edges is not large for $N=10$ and $N=11$. Similarly, the staggered magnetization increases when the ring width decreases.

Figure 2 shows how the density of states (DOS) of the $9_{A C}: N_{Z G}$ rings (the cases depicted in Fig. 1) varies with $N$. The density of states for the noninteracting (interacting) case is displayed by the black (purple) lines. In order to align the interacting and noninteracting spectra for easier comparison we subtracted $\Delta E$ for each interacting spectrum. Note that the density of states is spin degenerate, which is in accordance with Lieb's theorem. For $N=3$, magnetic order is not present, therefore the energy dependence of the density of states for the interacting and noninteracting systems coincide [see Fig. 2(a)]. The interacting and noninteracting electron case exhibit a small difference in the energy dependence of the DOS for rings with $N=4$ and $N=5$, which is shown in Figs. 2(b) and 2(c). As could be inferred from Fig. 1(c), the magnetization along the inner ring edge is rather small for these values of $N$. For larger $N$, the discrepancy between the DOSs for the interacting and noninteracting systems becomes larger, as demonstrated by Figs. 2(d)-2(f) for $N=9,10$, and 11. In all these cases, appreciable DOS for the noninteracting system is found around zero energy. Such a configuration becomes unstable in the presence of $e$-e interactions, which results in the appearance of an interaction gap.

In order to demonstrate how the shape of the outer boundary affects the distribution of the magnetic moments in the ring, we show in Fig. 3 the magnetization in the $13_{Z G}: N_{Z G}$ rings. It is apparent that the shape of the outer edge has a large effect on the localization of the magnetic moments on this boundary [compare Figs. 1(c) and 3]. It is clear that in the case of zigzag outer ring edge, the magnetization propagates much further into the ring.

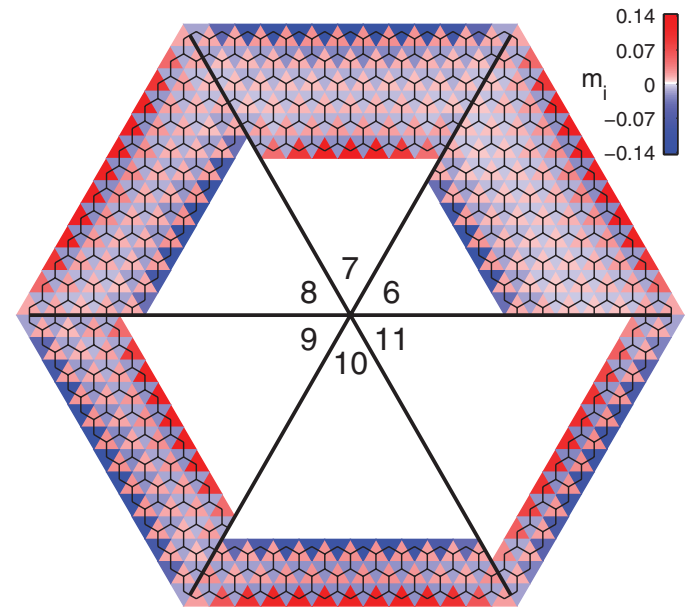

FIG. 3. (Color online) Distribution of magnetic moments in the $13_{Z G}: N_{Z G}$ rings for $N$ ranging from 6 to 11 .

Figure 4 displays how $\mu_{s}^{z}, m_{\max }$, and $\Delta E$ vary with the length of the side of the inner ring edge expressed by the number $N$. Along with the rings whose magnetic moment distributions were shown in Fig. 1(c) and Fig. 3, the case of a hexagonal graphene dot having a zigzag edge is also displayed in Fig. 4. Both the staggered magnetization $\mu_{s}^{z}$ and the energy shift $\Delta E$ increase with $N$, i.e., with the size of the inner ring, except for the extremely narrow $M_{Z G}$ : $N_{Z G}$ rings. Interestingly, the staggered magnetization in the hexagonal quantum dots does not exceed 0.02 , whereas for the $13_{Z G}: N_{Z G}$ ring it can reach almost up to 0.05 . The nearly twofold enlargement of the staggered magnetization could be accounted for by the double number of zigzag edges in the $M_{Z G}: N_{Z G}$ ring as compared to the $N_{Z G}$ graphene dot. On the other hand, most $9_{A C}: N_{Z G}$ rings exhibit larger staggered magnetization and all show larger maximum magnetic moment

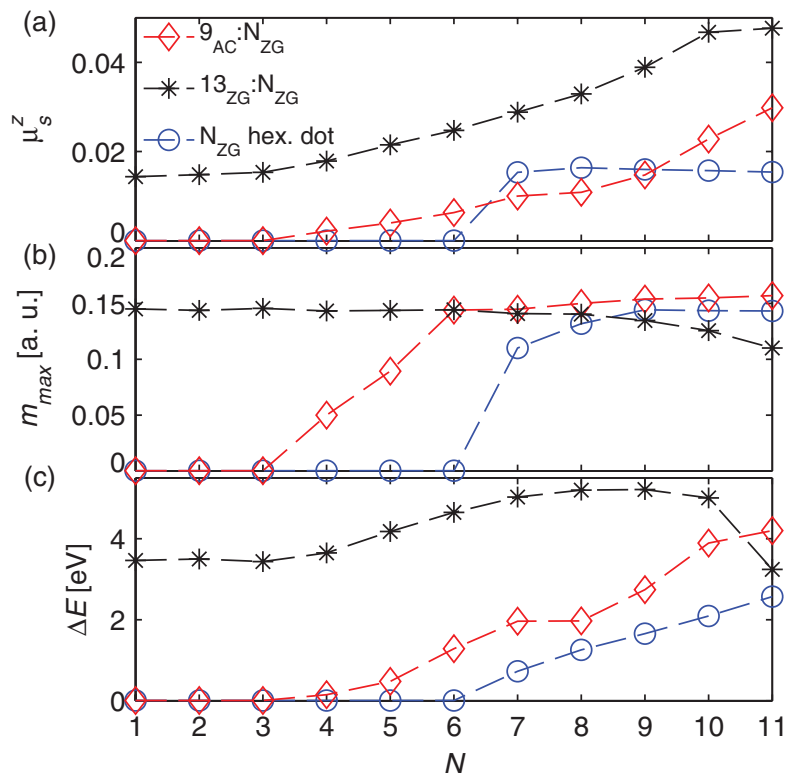

FIG. 4. (Color online) (a) Staggered magnetization $\mu_{s}^{z}$, (b) maximum moment $m_{\max }$, and (c) energy shift $\Delta E$ as they vary with the length of the side of the inner ring edge. 
than the hexagonal graphene dot. As a matter of fact, in hexagonal graphene dots the zero-energy orbitals which are localized along the adjacent zigzag sides of the edge are oriented toward each other, whereas inner zigzag edges in rings face away from each other. Hence, hybridization between the states of the two edges is larger in the former case than in the latter case. This is why $9_{A C}: N_{Z G}$ rings turn magnetic for shorter lengths of zigzag edges than hexagonal dots (four versus seven, respectively). The decrease of $m_{\max }$ with $N$ for $13_{Z G}: N_{Z G}$ is due to the more effective hybridization between the quasi-zero-energy states localized on the inner and outer edges of the ring when the ring width decreases. Hence, the electron energy shifts from the band of zero-energy states, and therefore magnetic ordering decays, which is manifested by a smaller $m_{\max }$ in the $13_{Z G}: 11_{Z G}$ ring than in the $11_{Z G}$ dot. The shapes of the $\Delta E(N)$ curves shown in Fig. 4(c) resemble the $\mu_{s}^{z}(N)$ and $m_{\max }(N)$ curves in Figs. 4(a) and 4(b).

In order to elucidate the difference between magnetic ordering in rings and dots, one may also analyze how the local density of states (LDOS) depends on the geometry of the structure. More specifically, the spatial distribution of the states close to zero energy determines how the magnetic moments evolve when the dimensions of the structures varies. In order to enhance the contribution of the low-energy states, we will compute the weighted LDOS (WLDOS): ${ }^{11}$

$$
W_{i}=\sum_{j} e^{-\beta E_{j}^{2}}\left|\phi_{j i}\right|^{2} .
$$

Here, $i$ indexes the lattice sites, $j$ labels the eigenstates, $\beta$ is the damping coefficient chosen as $1 / \sqrt{\beta}=0.1 \mathrm{eV}$, whereas $\phi_{j i}$ is the value of the probability amplitude of the $j$ th state at site $i$. Such defined WLDOS assumes that the contribution of the states with $\left|E_{j}\right|>0.1 \mathrm{eV}$ is negligible. The plots of the WLDOS in Fig. 5(a) illustrate how the edge states form when the inner $7_{Z G}$ hexagonal dot is cleaved out of the outer $7_{A C}$ hexagonal dot. The inner dot is separated from the ring by severing the bonds one by one between the dot and the ring. The number of severed bonds between the dot and the ring is explicitly shown in Fig. 5(a), and the dot edge is depicted by the blue line. The local sublattice imbalance accumulates quickly with the number of severed bonds, but no edge states emerge when the number of cut bonds is less than four. The edge states, which are depicted by red contours around the edge, are initially distributed evenly between the ring and the dot, but they extend more to the ring when the number of cut bonds increases.

To explore this finding in more detail, we show in Fig. 5(b) how the total WLDOS (full purple circles), which is the sum over the atomic sites in the dot (full blue circles) and the ring (empty red circles), varies with the number of severed bonds. Also, the DOS at zero energy is shown by the black triangles in Fig. 5(b). Notice that the variation of the WLDOS has a similar shape for each side of the ring's inner edge, and that the WLDOS displays step-like features. These steps arise because the imbalance between the two local sublattices, found at the ring and dot sides of the newly formed edge, are maximized when the formation of each side of the ring's inner edge is completed. The next side of the ring's inner edge contains the opposite sublattice imbalance, and therefore the states on this
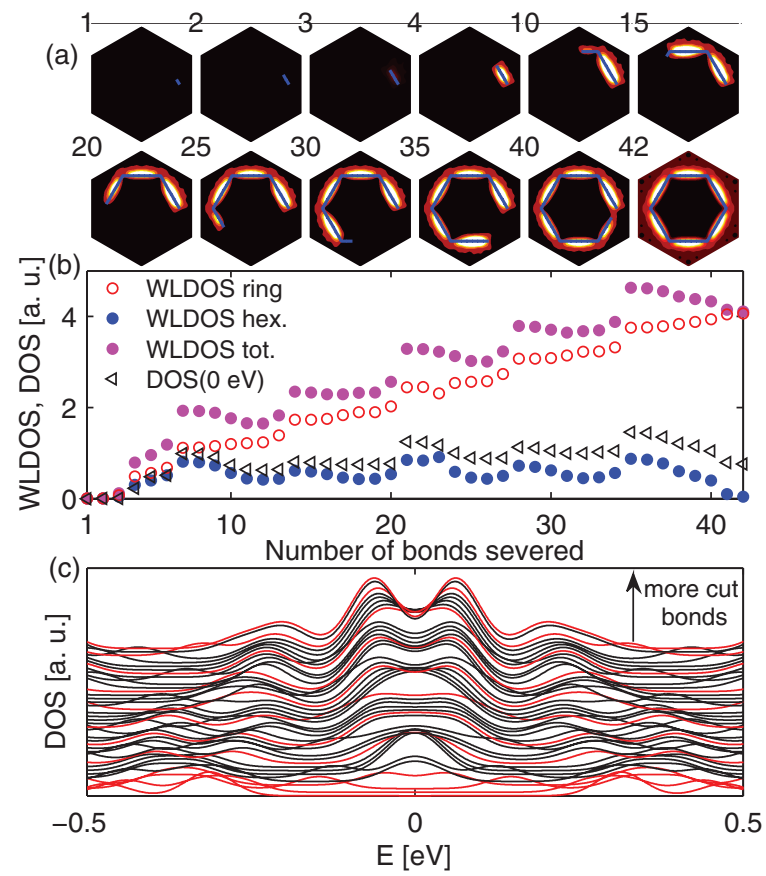

FIG. 5. (Color online) (a) Contour plot of WLDOS at several stages of the carving process forming the $7_{A C}: 7_{Z G}$ ring and the $7_{Z G}$ hexagonal dot; the number in the upper-left corner indicates the number of bonds cut. (b) Summed WLDOS in the ring, the dot, and in the whole structure as well as the zero-energy density of states versus the number of bonds cut. (c) Stacked plot of the density of states; red depicts the densities of the stages displayed in panel (a).

side hybridize with the states on the previous side, which leads to a decrease of WLDOS. ${ }^{11}$ Note that after the first edge has been cut the ring and the dot WLDOSs start deviating from each other more strongly. This is because the hybridization in the dot is stronger, as the edge states on adjacent segments hybridize inward and toward each other. In the ring part the edge states face away from each other and hybridize radially outward, hence the hybridization is weaker. This is why the WLDOS in the former case experiences a decline with the beginning of each new edge segment, while in the latter case the WLDOS keeps growing. The gradual increase of WLDOS for both cases near the end of each segment is related to the accumulation of the local sublattice imbalances. This pattern reappears with each new zigzag segment, with the exception of the last bond, which after being cut results in the separation of the two structures. By the end, the WLDOS in the ring is much larger than WLDOS in the dot, which accounts for the fact that the rings exhibit a larger maximum magnetic moment and staggered magnetization than the dots. Figure 5(c) shows a stacked plot of DOSs for each resulting structure. Plots are stacked from the bottom up, with each subsequent line corresponding to a structure with one more bond cut. DOSs for structures depicted in Fig. 5(a) are colored red. It shows that only features near zero energy evolve in a similar fashion as the WLDOSs during the separation of the ring and the dot. This justifies the damping of states higher than $0.1 \mathrm{eV}$ in calculating the WLDOS, as they are not artifacts of the edge forming between the ring and the dot. 


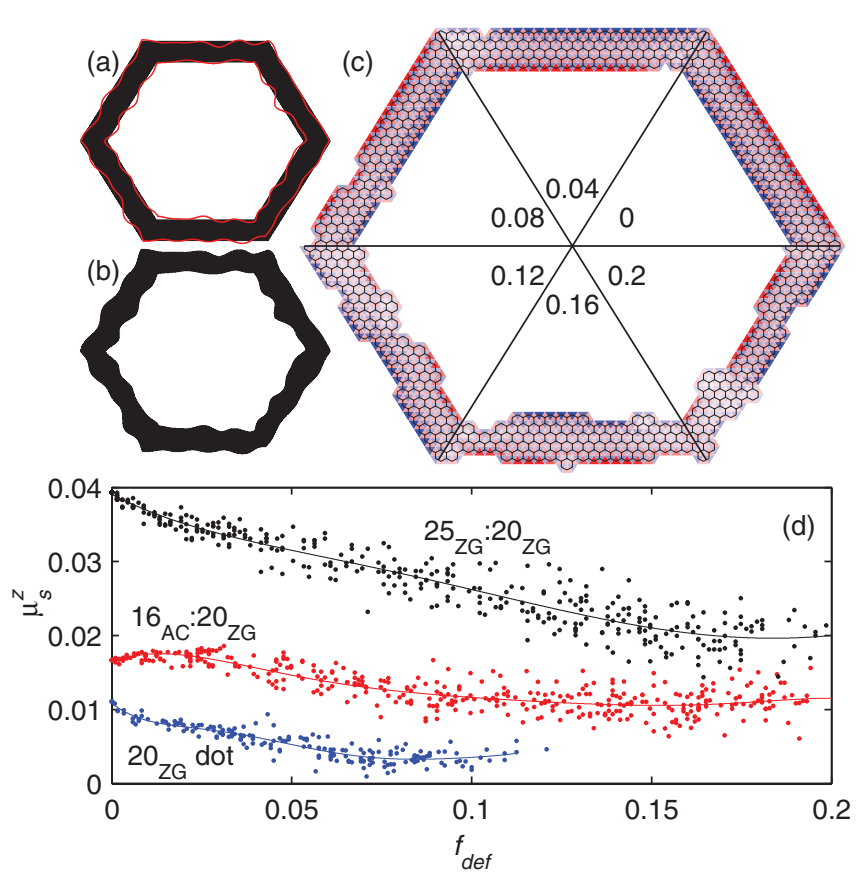

FIG. 6. (Color online) (a) Perfect edges (black region) are randomly perturbed (red lines) to produce a random set of defects. (b) Final outlook of the deformed ring. (c) Magnetic moments distributions in $25_{Z G}: 20_{Z G}$ ring for several values of $f_{\text {def. }}$ The scale is the same as in Fig. 3. (d) Staggered magnetization of an ensemble of randomly defected structures as a function of defect ratio for $25_{Z G}: 20_{Z G}$ ring (black dots), $16_{A C}: 20_{Z G}$ ring (red dots) ring, and $20_{Z G}$ hexagonal dot (blue dots). The polynomial fitting curves are added to guide the eye.

Finally, we examine the influence of the edge deformations on somewhat larger structures; namely, the $25_{Z G}: 20_{Z G}$ and $16_{A C}: 20_{Z G}$ rings and the $20_{Z G}$ dot. Larger structures are considered here because they can be deformed in a larger number of ways than smaller structures analyzed in the rest of the paper. Defects are induced by randomly deforming the polygons which outline the perfect structure as shown in Fig. 6(a). The amplitude of this deformation is itself a randomly selected number out of a specific range and the final structure is made up of all atoms that are enclosed by the deformed outline, ${ }^{31}$ which is shown in Fig. 6(b). In order to quantify the amount of defects, the defect ratio $f_{\text {def }}$ is defined as a fraction of the total number of the defects, which is a sum of the missing and the surplus sites, and the number of the sites in the original structure. The magnetic moment distributions in the $25_{Z G}: 20_{Z G}$ ring for a few values of $f_{\text {def }}$ are shown in Fig. 6(c). Also, variation of the staggered magnetization with the defect fraction for the $16_{A C}: 20_{Z G}$ and $25_{Z G}: 20_{Z G}$ rings and the $20_{Z G}$ dot is displayed in Fig. 6(d). For the $25_{Z G}: 20_{Z G}$ ring and the $20_{Z G}$ hexagon, $\mu_{s}^{z}$ decreases with defect fraction. This is expected, having in mind that the defects can only impair the conditions for magnetism in zigzag edges. On the other hand, for the $16_{A C}: 20_{Z G}$ ring, small random defects are more likely to make the larger outer edge magnetic than to make the smaller inner edge nonmagnetic. This explains the initial rise in $\mu_{s}^{z}$ for $f_{\text {def }}$ up to 0.02 .

In conclusion, we predict an antiferromagnetic phase in hexagonal graphene rings with zigzag inner edge within the mean-field Hubbard model. The distribution of magnetic moments is found to strongly depend on the type of outer edge, and larger antiferromagnetic order is found in rings than in hexagonal dots. Peculiar hybridization between the states of adjacent sides of the inner ring edge is found to lead to an increase of magnetization of rings with respect to dots. Also, the staggered magnetization and the maximum magnetic moment are found to be strongly influenced by the size and the shape of the rings. For wide rings, the maximum magnetic moment is largest when both the inner and outer edges are zigzag. But, as a consequence of the hybridization between the states of the two edges, the maximum magnetic moment in a ring with armchair outer edge exceeds the one for the zigzag outer edge when the ring width decreases. The staggered magnetization in both the hexagonal dots and the rings with zigzag outer edge is found to decrease faster than in the rings with armchair outer edge when the number of the edge defects increases.

This work was supported by the EuroGRAPHENE programme of the ESF (project CONGRAN), the Serbian Ministry of Education, Science, and Technological Development, and the Flemish Science Foundation (FWO-Vl). *marko.grujic@etf.bg.ac.rs

†milan.tadic@etf.bg.ac.rs

${ }^{\ddagger}$ francois.peeters@ua.ac.be

${ }^{1}$ K. S. Novoselov, A. K. Geim, S. V. Morozov, D. Jiang, Y. Zhang, S. V. Dubonos, I. V. Grigorieva, and A. A. Firsov, Science 306, 666 (2004).

${ }^{2}$ M. T. Ong and E. J. Reed, ACS Nano 6, 1387 (2012).

${ }^{3}$ M. F. El-Kady, V. Strong, S. Dubin, and R. B. Kaner, Science 335, 1326 (2012).

${ }^{4}$ T. J. Echtermeyer, L. Britnell, P. K. Jasnos, A. Lombardo, R. V. Gorbachev, A. N. Grigorenko, A. K. Geim, A. C. Ferrari, and K. S. Novoselov, Nat. Commun. 2, 458 (2011).

${ }^{5}$ Y. M. Lin, C. Dimitrakopoulos, K. A. Jenkins, D. B. Farmer, H. Y. Chiu, A. Grill, and Ph. Avouris, Science 327, 662 (2010).
${ }^{6}$ L. Britnell, R. V. Gorbachev, R. Jalil, B. D. Belle, F. Schedin, A. Mishchenko, T. Georgiou, M. I. Katsnelson, L. Eaves, S. V. Morozov, N. M. R. Peres, J. Leist, A. K. Geim, K. S. Novoselov, and L. A. Ponomarenko, Science 335, 947 (2012).

${ }^{7}$ O. V. Yazuev, Rep. Prog. Phys. 73, 056501 (2010).

${ }^{8}$ Y.-W. Son, M. L. Cohen, and S. G. Louie, Nature (London) 444, 347 (2006).

${ }^{9}$ J. Fernandez-Rossier and J. J. Palacios, Phys. Rev. Lett. 99, 177204 (2007).

${ }^{10}$ J. Fernandez-Rossier, Phys. Rev. B 77, 075430 (2008).

${ }^{11}$ J. J. Palacios, J. Fernandez-Rossier, and L. Brey, Phys. Rev. B 77, 195428 (2008)

${ }^{12}$ H. S. S. Ramakrishna Matte, K. S. Subrahmanyam, and C. N. R. Rao, J. Phys. Chem. C 113, 9982 (2009). 
${ }^{13}$ T. Enoki and K. Takai, Solid State Commun. 149, 1144 (2009).

${ }^{14}$ V. L. Joseph Joly, M. Kiguchi, S. J. Hao, K. Takai, T. Enoki,

R. Sumii, K. Amemiya, H. Muramatsu, T. Hayashi, Y. A. Kim, M. Endo, J. Campos-Delgado, F. López-Urías, A. Botello-Méndez, H. Terrones, M. Terrones, and M. S. Dresselhaus, Phys. Rev. B 81, 245428 (2010).

${ }^{15}$ A. Ney, P. Papakonstantinou, A. Kumar, N.-G. Shang, and N. Peng, Appl. Phys. Lett. 99, 102504 (2011).

${ }^{16}$ R. R. Nair, M. Sepioni, I-Ling Tsai, O. Lehtinen, J. Keinonen, A. V. Krasheninnikov, T. Thomson, A. K. Geim, and I. V. Grigorieva, Nat. Phys. 8, 199 (2012).

${ }^{17}$ Y. Wang, Y. Huang, Y. Song, X. Zhang, Y. Ma, J. Liang, and Y. Chen, Nano Lett. 9, 220 (2009).

${ }^{18}$ J. Hong, E. Bekyarova, P. Likang, W. A. de Heer, R. C. Haddon, and S. Khizroev, Sci. Rep. 2, 624, (2012).

${ }^{19}$ J. Kunstmann, C. Özdoğan, A. Quandt, and H. Fehske, Phys. Rev. B 83, 045414 (2011).

${ }^{20}$ O. V. Yazyev and M. I. Katsnelson, Phys. Rev. Lett. 100, 047209 (2008).

${ }^{21}$ M. Wimmer, I. Adagideli, S. Berber, D. Tománek, and K. Richter, Phys. Rev. Lett. 100, 177207 (2008).
${ }^{22}$ W. L. Wang, O. V. Yazyev, S. Meng, and E. Kaxiras, Phys. Rev. Lett. 102, 157201 (2009).

${ }^{23}$ M. Ezawa, Phys. E (Amsterdam, Neth.) 42, 703 (2010).

${ }^{24}$ X. Wei, M. Wang, Y. Bando, and D. Golberg, ACS Nano 5, 29162922 (2011).

${ }^{25}$ E. H. Lieb, Phys. Rev. Lett. 62, 1201 (1989).

${ }^{26}$ P. Potasz, A. D. Guclu, O. Voznyy, J. A. Folk, and P. Hawrylak, Phys. Rev. B 83, 174441 (2011).

${ }^{27} \mathrm{We}$ also include the constant part $U / 4-U / 2 \sum_{i, \sigma} n_{i, \sigma}$ in the Hamiltonian.

${ }^{28}$ T. O. Wehling, E. Şaşioğlu, C. Friedrich, A. I. Lichtenstein, M. I. Katsnelson, and S. Blügel, Phys. Rev. Lett. 106, 236805 (2011).

${ }^{29}$ H. Feldner, Zi Yang Meng, Andreas Honecker, Daniel Cabra, Stefan Wessel, and Fakher F. Assaad, Phys. Rev. B 81, 115416 (2010).

${ }^{30}$ K. Nakada, M. Fujita, G. Dresselhaus, and M. S. Dresselhaus, Phys. Rev. B 54, 17954 (1996).

${ }^{31}$ We remove all the dangling atoms which might appear after the edge is deformed. Also, we ensure that all the deformed structures have equal numbers of the A and B sublattice sites, thus our calculations always regarded the antiferromagnetic limit. 